\title{
Charge Asymmetry in the Brane World and Formation of Charged Black Holes
}

\author{
Herman J. Mosquera Cuesta ${ }^{1,2,3 *}$, André Penna-Firme ${ }^{1,4 \dagger}$, Abdel Pérez-Lorenzana ${ }^{1,5 \ddagger}$ \\ ${ }^{1}$ The Abdus Salam International Centre for Theoretical Physics, I-34100, Trieste, Italy \\ ${ }^{2}$ Centro Brasileiro de Pesquisas Físicas, Laboratório de Cosmologia e Física Experimental de Altas Energias \\ Rua Dr. Xavier Sigaud 150, 22290-180, RJ, Brasil \\ ${ }^{3}$ Centro Latinoamericano de Física (CLAF), Avenida Wenceslau Braz 173, Rio de Janeiro, RJ, Brasil \\ ${ }^{4}$ Universidade Federal do Rio de Janeiro (UFRJ), Faculdade de Educação, Av. Pasteur, 250, 22290-180, RJ, Brasil \\ ${ }^{5}$ Departamento de Física, Centro de Investigación y de Estudios Avanzados del I.P.N. \\ Apdo. Post. 14-740, 07000, México, D.F., México
}

(June, 2002)

\begin{abstract}
In theories with an infinite extra dimension, free particles localized on the brane can leak out to the extra space. We argue that if there were color confinement in the bulk, electrons would be more able to escape than quarks and than protons (which are composed states). Thus, this process generates an electric charge asymmetry on brane matter densities. A primordial charge asymmetry during Big Bang Nucleosynthesis era is predicted. We use current bounds on this and on electron disappearance to constrain the parameter space of these models. Although the generated asymmetry is generically small, it could be particularly enhanced on large densities as in astrophysical objects, like massive stars. We suggest the possibility that such accumulation of charge may be linked, upon supernova collapse, to the formation of a charged Black Hole and the generation of Gamma-Ray Bursts.
\end{abstract}

Extra dimensions could exist in nature and yet remain hidden to current experiments. Motivated by the old ideas of Kaluza and Klein and the modern string theory one has usually believed that such extra dimensions should be compact. Recently, however, it has been suggested the possibility that such extra dimensions can be rather infinite $[1-3]$ and still be hidden to our eyes. The explanation of why it is so comes from the concept of localization of particles [3-6] in a higher dimensional space (the bulk) around a fixed point that defines a four dimensional hypersurface (the brane). Thus, at low energy, observed particles would behave just as four dimensional fields. A simple realization of this scenario appears on five dimensional theories where the background space is given by the Randall-Sundrum (RS) metric [1]

$$
d s^{2}=e^{-2 k|y|} \eta_{\mu \nu} d x^{\mu} d x^{\nu}-d y^{2} ;
$$

where the parameter $k$ relates the fundamental five dimensional gravity scale, $M_{*}$, with the now effective Planck scale, $M_{P} \sim 10^{19} \mathrm{GeV}$, through $M_{*}^{3}=k M_{P}^{2}$.

The above metric induces an effective potential for gravitons that has the form of a volcano barrier [4] around the brane (located at $y=0$ ). The corresponding spectrum of gravitational perturbations has a massless bound state localized at the brane and a continuum of bulk modes with suppressed couplings to brane fields. The bound state is the true graviton that mediates the standard four dimensional gravity interaction. Bulk modes exchange induces on the brane deviations from the four dimensional Newton's law of gravity only at distances smaller than $k^{-1}$. Recent experiments that have tested gravity down to millimeters [7] require that $k \gtrsim 10^{-3} \mathrm{eV}$, which means $M_{*} \gtrsim 10^{8} \mathrm{GeV}$.

Massless scalar fields on RS background have a simi- lar spectrum, and so a bound state too [8]. Localization of fermions also takes place provided one has a domain wall on the background $[3,5,9]$. Finally, localization of gauge particles is also possible $[6,10]$. Last, however, according to Ref. [10] that we will follow heron, needs the existence of more extra flat dimensions compactified to small radii, $R \sim M_{*}^{-1}$. In such a setup there is a localized zero mode gauge boson, which alone satisfies the $4 \mathrm{D}$ Gauss law. Bulk modes introduce small corrections at short distances, though. The introduction of more compact dimensions, nevertheless, does not affect the model of localization of matter fields since all heavy modes, associated to the compact space, shall always be integrated out from the theory.

True localization, however, takes place only for massless fields $[9,11]$. In the massive case the bound state becomes metastable and able to leak into the extra space. Such a process appears as an apparent source for the violation of conservation laws in the effective four dimensional world. The associated escape rate can be calculated in specific models for localization of free fields [9]. Other interactions may also affect the escape rate by trying to keep the particles on the brane. So happens to quarks, provided there is confinement in color interactions along all dimensions [10].

As we argue along this letter, in such a scenario, the overall effect would be the generation of a tiny electric charge asymmetry, due to the escaping of electrons, which may be accumulated on large brane matter densities. For instance, out of the matter asymmetry on the early Universe. Current bounds on electron disappearance and on charge asymmetry at the Big Bang Nucleosynthesis (BBN) era can then be used to constrain the parameters of the model. We suggest that this mecha- 
nism could account for the formation of charged Black Holes (BH's), for instance after a supernova collapse. One possible implication of this would be the triggering of Gamma-Ray Bursts from vacuum polarization as proposed in Ref. [12].

Particle leakage and generation of charge asymmetry.In the models under consideration massive fields are actually metastable states. This happens because the mass lifts the bound state on the spectrum, whereas the continuum of modes remains unaffected, and thus, our formerly localized mode turns out to be not longer the lightest state. Indeed, there would be a non zero probability for the escaping of brane fields into the bulk $[9,10]$. One can check this by considering the localization mechanism of a massive fermion. We stress that the following arguments do not preclude the theory of having small extra compact dimensions. So, our results are consistent with the localization of gauge fields [10]. To be clear, we open a parenthesis to remain the reader how the localization of the photon works. In presence of $n$ extra flat dimensions, with coordinates $\theta_{i}$, the action for a photon gauge field, truncated at the zero mode level of $\theta$, has the form:

$$
S_{\gamma} \approx-\frac{1}{4} \int d^{4} x d y e^{-k n|y|} F_{\mu \nu} F^{\mu \nu}+\text { (heavy modes). }
$$

Hence the truely $y$-independent zero mode is normalizable, and thus it is localized at the brane. For fermions the presence of $\theta$ dimensions only rescale the normalization of the zero mode, so we can safely treat the problem as effectively five dimensional. Following Ref. [9], we consider a massive fermion, $\psi$, coupled to a domain wall background described by a scalar field, which in the thin wall approximation has the form $\phi(y)=v \operatorname{sgn}(y)$. The $\psi$ coupling to $\phi$ splits the chiral components of the four-component fermion such that only one of them gets localized around the wall. Thus, in order to give rise to a four dimensional mass term we are forced to introduce two five dimensional four-component fermions, $\psi_{1}$; $\psi_{2}$, which will develop left and right localized modes, respectively. For simplicity one accommodates those fields in an eight-component array of the form $\Psi=\left(\psi_{1}, \psi_{2}\right)$. With this notation the respective action can be written as [9]

$$
S=\int d^{4} x d y \sqrt{g} \bar{\Psi}\left(i \gamma^{a} \nabla_{a}+\phi \sigma_{1}+\mu \sigma_{2}\right) \Psi ;
$$

where $\nabla_{a}$ is the spinor covariant derivative with respect to the five dimensional metric $g_{a b}$ and $\sigma_{i}$ are the Pauli matrices acting on the $\psi_{1}, \psi_{2}$ space. The general form of the Dirac equation in terms of chiral components $\Psi_{L, R}$ is then given by

$$
e^{k|y|} \gamma^{\mu} p_{\mu} \Psi_{L, R} \pm \partial_{5} \Psi_{R, L}-\left(\phi(y) \sigma_{1}+\mu \sigma_{2}\right) \Psi_{R, L}=0 .
$$

This equation has a continuum of massive modes [9] that starts at $m=0$, for $m^{2}=p_{\mu} p^{\mu}$, and a localized massive mode (that has radiation boundary conditions at $y \rightarrow$ $\pm \infty$ ) for which the mass eigenvalue is complex: $m=$ $m_{0}+i \Gamma$, where the physical mass $m_{0}=(1-k / 2 v) \mu$. The imaginary part on $m$ reflects the metastable nature of the state. $\Gamma$ is then interpreted as the 'life time' of the particle, from the brane point of view.

In the case where $m_{0} \ll k<v$ the probability for escaping goes as

$$
\Gamma=m_{0} \cdot\left(\frac{m_{0}}{2 k}\right)^{2 v / k-1} \cdot \frac{\pi}{\left[\Gamma\left(v / k+\frac{1}{2}\right)\right]^{2}},
$$

This case will probe to be the most interesting one on our discussion below. By taking $k \gg m_{e}=5.11 \cdot 10^{-4} \mathrm{GeV}$, we state our interest to models where $M_{*}>10^{12} \mathrm{GeV}$. The theory of escaping matter is thus described by three parameters: the mass of the particle, $m_{0}$; the vacuum parameter, $v$; and the fundamental scale, $M_{*}$, encoded in the parameter $k$.

For charged particles, their lost into the bulk account for an apparent violation of the conservation of charge [10], say for instance through the process $e^{-} \rightarrow$ nothing. The picture is intrinsically a higher dimensional problem, and so, four dimensional Gauss law is not longer valid, though causality is conserved. In fact, a particle moving with a trajectory perpendicular to the brane follows the world line: $y(t)=\ln \left(1+k^{2} t^{2}\right) / 2 k$. It can be seen as a charged current which induces an electromagnetic field on the brane that dies away causally with time [10]. Indeed, out of the light cone $(|\vec{r}|>t)$ the Coulomb potential of the charge remains: $\phi(\vec{r}) \sim 1 / r$, whereas deep in the light cone the electric field gradually disappears as $E \sim r / t^{3}$. This problem has a gravitational analogous studied in Refs. [11,13].

While escaping away is certainly possible for fundamental particles, it is not so simple for composed states. These do not leak away from the brane as a whole so easily since this needs the simultaneous leakage of all components, which substantially reduces the escaping probability. For instance, for protons one naively gets a negligible escape rate: $\Gamma_{P} \sim m_{P} P_{u} P_{u} P_{d} \cdots$, with $P_{u, d}$ the escaping probability of quark components.

In hadrons, confinement of color interactions may prevent the escape of a single colored component [10]. It is believed that due to confinement the color flux does not spread around as gravity and electromagnetism do. It rather forms flux tubes connecting quarks that act as an unidimensional strong attractive force that grows with distance, in spite of the orientation of quarks on space. It is then natural to expect that color will also be confined along the fifth dimension, thus, making the trapping on the brane even stronger. This also forbids processes that may induce proton decay, and the sudden creation of colored composed states from uncolored ones. This asymmetric nature between electrons and protons translates into the only disappearance of electrons from the 
brane, and induces an excess of charge (in the form of protons) in any actual brane matter density (otherwise neutral). Notice, however, that if color were not confined on the bulk, a charge asymmetry will be generated any way due to the different charge (mass) of electrons and quarks, but we will not discuss this case in here. Next, let us confront this result with some known current bounds that in turn will constrain the $v-M_{*}$ space parameter.

Bounding the parameter space.- The first straightforward constraint comes from the bound on electron disappearance, which is about $\tau_{e \rightarrow \text { nothing }}>4.2 \cdot 10^{24} \mathrm{yr}$ [14]. This implies that escape rate of electron should be

$$
\Gamma \lesssim \Gamma_{\text {exp }} \equiv 1 / \tau_{e \rightarrow \text { nothing }}=4.96 \cdot 10^{-57} \mathrm{GeV} .
$$

A second phenomenological constraint comes from the fact that the fundamental scale is supposed to be the largest scale on the theory, and thus $v \leq M_{*}$. In Fig. 1, we have plotted the curves that saturate both the bounds. The allowed region in the parameter space for any viable model lies between both the curves and well within the region where $k<v$. In fact, the lower border can be described by a (phenomenological) power law relationship between $v$ and $M_{*}$ of the form: $v \approx\left(M_{*} / M\right)^{1.91} M_{*}>k$. Hence, one concludes that all scenarios where $k \geq v$ are excluded on the basis of current experimental bounds.

Future improvements on present limits on electron disappearance would lift the lower border on Fig. 1. The effect, however, may be very small even for substantial improvements. For instance, an increment of nine orders of magnitude on the bound, that would render $\tau_{e \rightarrow \text { nothing }}>10^{33} \mathrm{yr}$, would hardly change the allowed region in parameter space as it is clear from Fig. 1, where the effect of this hypothetical bound is also depicted.

Charge asymmetry at BBN era.- On the early Universe, a primordial charge asymmetry can be induced out of the primordial matter-antimatter asymmetry. The point is that in a balanced Universe, where electrons and positrons are equal in number, no charge asymmetry can be induced, since the escape rate has no dependence on the sign of the charge. However, since there is in fact an initial matter to antimatter asymmetry per photon of order $\Delta \eta_{m} / \eta_{\gamma} \sim 10^{-10}$, some deficit of charge can indeed be generated. Though the above asymmetry is originally in the form of baryons, some processes (as sphalerons) are expected to generate a similar lepton asymmetry. Thus, we would be looking into an initially neutral Universe where most of charged particles are equilibrated by an antiparticle, except by a very little amount of protons, as much as $\Delta \eta_{m}$, that are compensated only by electrons. Out of these initial $\Delta \eta_{m}$ free electrons, some will leak out from the brane, such that at the BBN era the remaining $\eta_{B B N}^{e}=\Delta \eta_{m} \cdot \exp \left(-\Gamma \tau_{B B N}\right)$ electrons will not balance the charge equilibrium. The density of charged particles in excess per photon would be:
$\Delta q_{B B N}=\left(\frac{\Delta \eta_{m}}{\eta_{\gamma}}\right)\left(1-e^{-\Gamma \tau_{B B N}}\right) \approx 10^{-10} \cdot \Gamma \tau_{B B N}$.

A net charge density on early Universe would produce large scale relic electric fields. Certainly in a truly homogeneous space the presence of a uniform charge density $\Delta q_{B B N}$ cannot give rise to any field. However, early Universe was not really homogeneous, otherwise not even structure formation could be possible. Indeed, there was a primordial inhomogeneity of order $\delta \rho / \rho \sim 10^{-5}$. Such fields, might be the seeds for the observed galactic and extragalactic magnetic fields, but we will not address this possibility here. These relic fields may also generate observable cosmic ray anisotropies. Current limits impose the bound [15] $\Delta q \lesssim 10^{-39}$. This gives, however, a weaker bound for the escape rate than the one already mentioned above. In fact one gets $\Gamma \lesssim 10^{-54} \mathrm{GeV}$ $\left(\tau_{e} \gtrsim 10^{21} \mathrm{yrs}\right)$. A stringent bound is obtained from the effect of a background charged Universe on primordial Helium production [16], which gives $\Delta q \lesssim 10^{-43}$. That implies

$$
\Gamma \lesssim \Gamma_{B B N}=6.58 \cdot 10^{-58} \mathrm{GeV} .
$$

This also means an enhancement on the electron disappearance bound by a factor of seven. The curve on the parameter space associated to the saturation of this last bound has been also plotted on Fig. 1, though it does not affect the previous picture at all.

Accumulation of charge on Stellar Objects.- Though the charge asymmetry induced by the leakage of charge might be too small as to be directly detected in terrestrial laboratories, it could be relevant in longevous astrophysical objects which contain a large number of particles. In order to estimate this asymmetry for dense media one has to consider the presence of internal forces that keep those systems all together in equilibrium in the empty interstellar space. The attractive interaction that compensates the internal pressure comes mainly from the selfgravitational interaction of the system. This attractive force will also tend to keep the particles on the brane, where the source is. From the five dimensional point of view the effect of these internal forces will be to locally lift the trapping barrier, thus, effectively reducing the escape rate. One can account for this effect by introducing a phenomenological function, $x(\vec{r})$, to locally measure the amount of time along which the particle behaves as effectively free, and able to escape. Thus, one can writes at zero order the particle density as $\eta(\vec{r}, t) \approx \eta(\vec{r}) e^{-x(\vec{r}) \Gamma t}$. From this equation one notices that the quantity $x \Gamma$ has the physical meaning of being the effective escape rate, as modified by the presence of interactions. So, $x$ plays the role of a structure factor that encodes the internal forces of the medium at which the particle is subjected. Now, as the total number of remaining electrons at any given time is given by $N_{e}(t)=\int d V \eta(\vec{r}, t)$, and due to 
the smallness of $\Gamma$, the induced charge asymmetry can be expressed in the form:

$$
\Delta q(t) \equiv \frac{N_{p}-N_{e}(t)}{N_{e}(0)}=1-\frac{N_{e}(t)}{N_{e}(0)} \approx \bar{x} \Gamma t ;
$$

where $\bar{x}$ indicates the volume average of $x$ that becomes the actual phenomenological parameter, yet to be calculated for realistic cases. It is expected that for most (not so dense) media $\bar{x} \approx 1$, however, it may be important for tightly bound systems as neutron stars.

Let us now consider a typical star to estimate how much of asymmetry it can accumulate along its life, $t_{*}$, which may be almost as large as the age of the universe. Thus, taking $t_{*}=10^{10}$ yr into Eq. (6), combined with the bounds on Eqs. (4) and (5) we get

$$
\Delta q\left(t_{*}\right) \lesssim 10^{-14,-16} .
$$

Charged Black Holes and Gamma-Ray Bursts- A tiny charge asymmetry would generate small electromagnetic fields which can be substantially magnified [17] by some astrophysical processes such as supernova core collapse. The presence of a non zero total charge on stars progenitor of supernovae could give rise to other fundamental phenomena like the ulterior formation of charged Black Holes. The origin of charged BH's has been a long standing puzzle in relativistic astrophysics after the derivation of Reissner-Nordström solution of Einstein's equations. Thence, this brane world inspired charge-leaking mechanism provides a natural explanation for their origin upon stellar evolution of massive stars, thus, solving such an enigma.

An interesting consequence of all this could be the generation of Gamma-Ray Bursts (GRBs) as already suggested in Ref. [12]. However, the open point is whether particle leakage can account for the required charge asymmetry to switch on such a process. The answer to this question appears to be on the positive. According to Ref. [12], vacuum polarization occurring during the formation phase of a Reissner-Nordström Black Hole may generate GRBs, provided that the net charge-to-mass ratio, $\xi=Q /(M \sqrt{G})$; be of the order 0.01 to one. The limit $\xi=1$ corresponds to a critical charged Black Hole. Nevertheless, it is likely that even smaller values of $\xi$ could still render workable the quoted mechanism. In any case, the above condition translates into an effective charge asymmetry per nucleon in the collapsing matter: $\Delta q_{B H} \approx \xi 10^{-18}$. This value is at least two orders of magnitude smaller than the upper bound on Eq. (7), and thus, it can indeed be provided by the charge leakage. Moreover, for generating such an asymmetry, one concludes that the electron has to disappear from the brane on a time, $\tau_{e}$, within the range

$$
4.2 \cdot 10^{24} \mathrm{yr} \lesssim \tau_{e} \lesssim 2.08 \xi^{-1} \cdot 10^{28} \mathrm{yr} .
$$

The allowed region in the parameter space that is consistent with these bounds is yet very narrow, thus, pointing towards a very specific class of models. Indeed, even for a $\xi \sim 10^{-5}$ that gives $\tau_{e} \lesssim 10^{33} \mathrm{yr}$; one gets the same narrow strip already depicted in Fig. 1 within current and hypothetical future limits.

Summarizing- In brane world models massive fundamental states become metastable with a non zero probability for escaping away into the bulk. Nevertheless, confinement of color interactions will prevent the leakage of quarks, while protons have a negligible scape rate. Therefore, only electrons would be effectively missed, and so, unbalancing the neutralness of brane matter densities. As a result modest charge asymmetries can be generated on the early Universe and in stellar objects, which may have observable effects. We confronted this result with current bounds on electron disappearance and relic electric charge at BBN era, and consequently constrained the parameters of the brane world model. We have suggested the possibility that supernova collapse of massive stars may indeed form charged Black Holes. We shown that the charge of such a Black Hole can be of the right order as to trigger GRBs via vacuum polarization. There is a non zero parameter space consistent with this picture that also points towards an electron disappearance rate non far from current experimental limits.

We thank A. Mazumdar for useful conversations. The work of APF was supported by a grant of Conselho Nacional de Desenvolvimento Científico e Tecnológico, CNPq-Brasil. HJMC thanks Fundação de Amparo à Pesquisa do Estado do Rio de Janeiro (FAPERJ) for a grant-in-aid.

[1] L. Randall, R. Sundrum, Phys. Rev. Lett. 83, 4690 (1999); M. Gogberashvili, hep-ph/9812296.

[2] N. Arkani-Hamed, et al., Phys. Rev. Lett. 84, 586 (2000).

[3] V.A. Rubakov, M.E. Shaposnikov, Phys. Lett. B125, 136 (1983).

[4] J. Likken, L. Randall, JHEP 0006, 014 (2000).

[5] R.Jackiw, C.Rebbi, Phys. Rev. D13, 3398 (1976).

[6] G. Dvali, M. Shifman, Phys. Lett. B396, 64 (1997).

[7] C.D. Hoyle, et al., Phys. Rev. Lett. 86, 1418 (2001).

[8] B. Bajc, G. Gabadadze, Phys. Lett. B474, 282 (2000); S.B. Giddings, E. Katz, L. Randall, JHEP 0003, 023 (2000).

[9] S.L. Dubovsky, V.A. Rubakov and P.G. Tinyakov, Phys. Rev. D 62, 105011 (2000).

[10] S.L. Dubovsky, V.A. Rubakov and P.G. Tinyakov, JHEP 0008, 041 (2000).

[11] R. Gregory, V.A. Rubakov, and S.M. Sibiryakov, Class. Quantum Grav. 17, 4437 (2000).

[12] R. Ruffini, et al., Astrophys. J. 555, L107 (2001).

[13] S.B. Giddings, E. Katz, J. Math. Phys. 42, 3082 (2001).

[14] P. Belli, et al., Phys. Lett. B460, 236 (1999).

[15] S. Orito, M. Yoshimura, Phys. Rev. Lett. 54, 2457 (1985). 
[16] E. Masso, F. Rota, astro-ph/0201248.

[17] S. L. Shapiro, S. A. Teukolsky, White dwarfs, black holes and neutron stars: The physics of compact objects (Wiley \& Sons, New York, 1983).

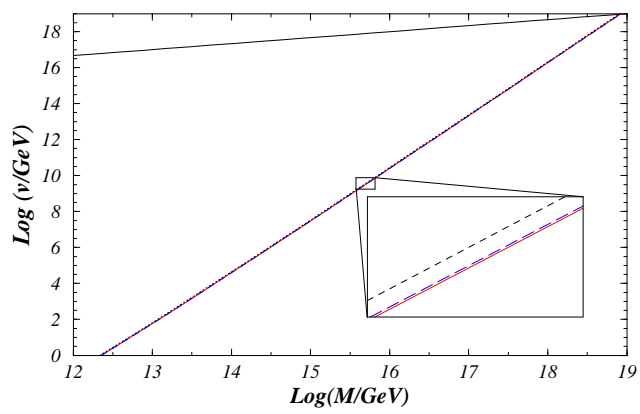

FIG. 1. Allowed parameter space for brane world models. Upper line stands for $v=M_{*}$. Lower line is the limit imposed by $\Gamma_{\text {exp }}$ (continuous line) and $\Gamma_{B B N}$ (dashed line). Dotted line on the blown up zone pictures an hypothetical future limit on electron disappearance of about $10^{33} \mathrm{yr}$. 